\title{
An Upper Bound for Functions of Estimators in High Dimensions
}

\author{
Mehmet Caner* Xu Han ${ }^{\dagger}$
}

August 7, 2020

\begin{abstract}
We provide an upper bound as a random variable for the functions of estimators in high dimensions. This upper bound may help establish the rate of convergence of functions in high dimensions. The upper bound random variable may converge faster, slower, or at the same rate as estimators depending on the behavior of the partial derivative of the function. We illustrate this via three examples. The first two examples use the upper bound for testing in high dimensions, and third example derives the estimated out-of-sample variance of large portfolios. All our results allow for a larger number of parameters, $p$, than the sample size, $n$.
\end{abstract}

Keywords and phrases: Many assets, many restrictions, Lasso.

\footnotetext{
*North Carolina State University, Nelson Hall, Department of Economics, NC 27695. EMAIL: MCANER@NCSU.EDU.

${ }^{\dagger}$ City University of Hong Kong, 83 Tat Chee Avenue, KL, Hong Kong S.A.R. Email: XUHAN25@CITYU.EDU.HK
} 


\section{Introduction}

The delta method is one of the most widely used theorems in econometrics and statistics. It is a very simple and a useful idea. It can provide limits for complicated functions of estimators as long as the function is differentiable. The limit of the function of estimators can be obtained from the limit of the estimators, with the same rate of convergence. In the case of finite-dimensional parameter estimation, since the derivative at the parameter value is finite, rates of convergence of both estimators and function of estimators are the same.

In the case of high dimensional parameter estimation, we show that this is not the case, and the rates of convergence may change. We show that the structure of the derivative of the function is the key. An upper bound random variable is provided for the functions of estimators in high dimensions. We show this upper bound on functions of estimators may converge faster, slower, or at the same rate as estimators. Even though a new delta theorem is not provided, this upper bound can get the rate of convergence of functions of estimators. For example, the variance of a portfolio is a quadratic function of the portfolio weight. Our theorem implies that the convergence rate of the portfolio variance is slower than that of the estimated weight when the number of assets is diverging (see Example 3 in Section 3). From now on we denote the number of assets as $p_{n}$ since they grow with sample size.

Our result is useful when the number of parameters $p_{n}$ is larger than the sample size $n$, where $p_{n}$ grows with $n$. The reason is that proofs in high dimensional problems

usually depend on knowing the rate of convergence of the bound, which may be a lasso-type estimation, oracle inequality, or problems in high dimensional portfolio 
analysis.

After the main theorem, we illustrate our point in three examples: first by examining a linear function of estimators that is heavily used in econometrics, second a new debiased lasso type of estimator, and third by analyzing the out-of-sample variance of a large portfolio of assets in finance.

Section 2 provides our theorem. Section 3 has three examples. Section 4 provides a discussion of how the results may be tied to nonparametric analysis and many weak instrument asymptotics. Appendix shows the proof and provides more examples.

\section{Upper Bound}

Let $\beta_{0}=\left(\beta_{1,0}, \cdots, \beta_{p_{n}, 0}\right)^{\prime}$ be a $p_{n} \times 1$ parameter vector with an estimator $\hat{\beta}=$ $\left(\hat{\beta}_{1}, \cdots, \hat{\beta}_{p_{n}}\right)^{\prime}$. Define a function $f(), f:. K \subset R^{p_{n}} \rightarrow R^{m}$. To be specific $m$ is a constant unless noted otherwise.

We provide two conditions for our theorem. First, denote a column vector of $p_{n}$ zeros by $0_{p_{n}}$. Let $f_{d}($.$) represent the m \times p_{n}$ matrix of derivatives.

Condition C1. For all $h \neq 0_{p_{n}}$, and $h$ is a $p_{n} \times 1$ vector

$$
\lim _{h \rightarrow 0} \frac{\left\|f\left(\beta_{0}+h\right)-f\left(\beta_{0}\right)-f_{d}\left(\beta_{0}\right) h\right\|_{2}}{\|h\|_{2}}=0
$$

where $\|.\|_{2}$ is the Euclidean norm for a generic vector, and $f_{d}\left(\beta_{0}\right)$ is an $m \times p_{n}$ matrix, whose $(i, j)$ th cell consists of $\partial f_{i} / \partial \beta_{j}$ evaluated at $\beta_{0}$, for $i=1, \cdots, m$, $j=1, \cdots, p_{n}$.

Second, define the rate of convergence of an estimator as $r_{n}$, a positive sequence 
in $n$, and when $n \rightarrow \infty, r_{n} \rightarrow \infty$.

Condition C2.

$$
r_{n}\left\|\hat{\beta}-\beta_{0}\right\|_{2}=O_{p}(1) .
$$

Condition $\mathrm{C} 1$ is a high-level assumption that shapes our function of interest. It restricts the function $f$ to be differentiable. Hence, $\mathrm{C} 1$ rules out continuous functions that are non-differentiable at $\beta_{0}$. Condition $\mathrm{C} 2$ gives a convergence rate for the estimator of interest. Several examples about $\hat{\beta}$ and $r_{n}$ that satisfy $\mathrm{C} 2$ are provided in Section 3.

The following theorem is the main theoretical result. Given the convergence rate of the high-dimensional estimator, it provides an upper bound (random variable) for the estimation error of functions of the high-dimensional estimator. This is useful in econometric theory since it can give us an idea about what the rate of convergence of functions of estimators might be. Let $k_{n}$ and $d_{n}$ be positive sequences in $n$ so that both $k_{n} \rightarrow \infty$ and $d_{n} \rightarrow \infty$ as $n \rightarrow \infty$. Take a generic matrix, $D$, of dimensions $m \times p_{n}:\|\mid D\|_{2}$ denotes the Frobenius norm of the matrix $D$ :

$$
\|\mid D\|_{2}=\sqrt{\sum_{i=1}^{m}\left\|d_{i}\right\|_{2}^{2}},
$$

where $d_{i}$ is a $p_{n} \times 1$ vector, and its transpose $d_{i}^{\prime}$ is the $i$ th row of $D$.

Theorem 2.1. Let Conditions $C 1$ and $C 2$ hold, and $C>0$ be a universal constant. Assume that $\left\|\left|f_{d}\left(\beta_{0}\right) \|\right|_{2}>0\right.$ 
a) If $\left\|\left|f_{d}\left(\beta_{0}\right) \|\right|_{2}=C\right.$, then

$$
\left\|f(\hat{\beta})-f\left(\beta_{0}\right)\right\|_{2} \leq L_{n}=O_{p}\left(\frac{1}{r_{n}}\right) .
$$

b) If $\left.\left\|f_{d}\left(\beta_{0}\right)\right\|\right|_{2}=C k_{n}$, then

$$
\left\|f(\hat{\beta})-f\left(\beta_{0}\right)\right\|_{2} \leq L_{n}=O_{p}\left(\frac{k_{n}}{r_{n}}\right) .
$$

c) If $\left\|\left|f_{d}\left(\beta_{0}\right) \|\right|_{2}=\frac{C}{d_{n}}\right.$, then

$$
\left\|f(\hat{\beta})-f\left(\beta_{0}\right)\right\|_{2} \leq L_{n}=O_{p}\left(\frac{1}{d_{n} r_{n}}\right)+o_{p}\left(\frac{1}{r_{n}}\right)=o_{p}\left(\frac{1}{r_{n}}\right) .
$$

Remarks. 1. The theorem does not provide a limit for functions of estimators, so this is not the delta theorem.

2. Part (a) shows under what conditions we can get the same rate of convergence for the functions of estimators compared with the rate of convergence of $\hat{\beta}-\beta_{0}$. Example 2 illustrates this point.

3. Part (b) shows that the $l_{2}$ norm of the partial derivative function may change with the dimension of the parameter vector. When $r_{n}>k_{n}$, and $k_{n} \rightarrow \infty$, then the upper bound $L_{n}$ converges at a slower rate than estimators of parameters. With $r_{n} / k_{n} \rightarrow 0$, then the upper bound is diverging which tells us that the function of estimators may diverge, too.

4. Part (c) shows that the function of the estimators converges to zero in probability faster than the rate of convergence of estimators $r_{n}$. 
5. Theorem 2.1 also holds with $l_{1}$ and $l_{\infty}$ norms. These new norm results can be shown when Conditions $\mathrm{C} 1$ and $\mathrm{C} 2$ hold in $l_{1}$ and $l_{\infty}$ norms. We discuss this in Part A of the Appendix.

\section{$3 \quad$ Examples}

We now provide three examples that highlight the contribution. The first one is related to the linear functions of estimators, the second one considers the Debiased Conservative Lasso (DCL) of Caner and Kock (2018), and the third one is related to the out-of-sample variance of large portfolios.

\section{Example 1.}

This example considers lasso, which is one of the benchmark methods in machine learning. It is a penalized least squares estimator with $l_{1}$ penalty. The penalty induces sparsity in the model, which can prevent overfitting (Hastie, Tibshirani, Friedman, 2009, Tibshirani, 1996).

Let us denote $\beta_{0}$ as the true value of vector $\left(p_{n} \times 1\right)$ of coefficients. The number of the true nonzero coefficients is denoted by $s_{0}$, and $s_{0}>0$. A simple linear model is:

$$
y_{t}=x_{t}^{\prime} \beta_{0}+u_{t}
$$

for $t=1, \cdots, n$. For simplicity, we assume that $u_{t}$ are iid errors with zero mean and finite variance and $x_{t}$ is a set of $p_{n}$ deterministic regressors. The iid assumption on $u_{t}$ is to keep our illustration simple and the following result still holds under more general assumptions on $u_{t}$ and $x_{t}$ as shown in Assumption 1 of Caner and Kock 
(2018).

The lasso estimator is defined as

$$
\hat{\beta}=\operatorname{argmin}_{\beta \in R^{p_{n}}}\left[\sum_{t=1}^{n} \frac{\left(y_{t}-x_{t}^{\prime} \beta\right)^{2}}{n}+2 \lambda_{n} \sum_{j=1}^{p_{n}}\left|\beta_{j}\right|\right],
$$

where $\beta_{j}$ is the $j$ th element of $\beta, \lambda_{n}$ is a positive tuning parameter, and it is established that $\lambda_{n}=O\left(\sqrt{\frac{\log p_{n}}{n}}\right)$. Corollary 6.14 or Lemma 6.10 of Buhlmann and van de Geer (2011) shows that, for lasso estimators $\hat{\beta}$, with $p_{n}>n$

$$
r_{n}\left\|\hat{\beta}-\beta_{0}\right\|_{2}=O_{p}(1)
$$

where

$$
r_{n}=\sqrt{\frac{n}{\log p_{n}}} \frac{1}{\sqrt{s_{0}}}
$$

Given (3.3), we may be interested in the large sample behavior of $D\left(\hat{\beta}-\beta_{0}\right)$, where $D$ is an $m \times p_{n}$ matrix. The $D$ matrix can be thought of putting restrictions on $\beta_{0}$. We want to see whether $D\left(\hat{\beta}-\beta_{0}\right)$ has a different rate of convergence from $\hat{\beta}-\beta_{0}$. From our Theorem 2.1(a), it is clear that $f_{d}\left(\beta_{0}\right)=D$. Basically in the case of inference, this matrix and the vectors show how many of $\beta_{0}$ will be involved with the restrictions. If we want to use $s_{0}$ elements in each row of $D$ to test $m$ restrictions, then $\|\mid D\|_{2}=O\left(\sqrt{s_{0}}\right)$. Note that this corresponds to using $s_{0}$ elements in $\beta_{0}$ for testing $m$ restrictions. In other words, if $k_{n}=s_{0}$ and $s_{0} \rightarrow \infty$ as $n \rightarrow \infty$, then Theorem 2.1(b) implies that

$$
L_{n}=O_{p}\left(\frac{\sqrt{s_{0}}}{r_{n}}\right) .
$$


This means that even with a fixed number of $m$ restrictions, the upper bound random variable has a slower rate of convergence than the estimators, so it is possible that functions of estimators also converge slower to a limit.

Remark. In high dimensions, a common assumption is to impose $\left\|d_{i}\right\|_{2}=1$. See, for example, Caner, Han and Lee (2018) and Caner and Kock (2018). In that case,

$$
\|\mid D\|_{2}=\sqrt{m}
$$

where $m$ is fixed, but $p_{n}$ is growing with $n$. The rate of convergence of $D\left(\hat{\beta}-\beta_{0}\right)$ will be still $r_{n}$, which is in (3.4). Thus, there will be no slowdown of the rate of convergence and this is a sharp rate since $\left.\left\|\left|f_{d}\left(\beta_{0}\right)\left\|\left.\right|_{2}=\right\|\right| D\right\|\right|_{2}=\sqrt{m}$. This is not an upper bound on $f_{d}\left(\beta_{0}\right)$.

Example 2. Another estimator that is recently analyzed in the context of $p_{n}>n$ is the DCL of Caner and Kock (2018). Consider the model in example 1 above in the matrix form:

$$
Y=X \beta_{0}+u
$$

where $Y$ is an $n \times 1$ vector, $X$ is an $n \times p_{n}$ matrix, $\beta_{0}$ is a $p_{n} \times 1$ vector that consists of $s_{0}$ nonzero parameters, $u$ is an $n \times 1$ error vector. $\hat{\beta}_{C L}$ is the conservative lasso estimator defined as

$$
\hat{\beta}_{C L}=\operatorname{argmin}_{\beta \in R^{p_{n}}}\left\{\|Y-X \beta\|_{2}^{2} / n+2 \lambda_{n} \sum_{j=1}^{p_{n}} \hat{w}_{j}\left|\beta_{j}\right|\right\},
$$

where $\hat{w}_{j}=\lambda_{n} / \max \left(\left|\hat{\beta}_{j}\right|, \lambda_{n}\right), \max (a, b)$ chooses maximum of two elements $a$ or $b$, 
and $\hat{\beta}_{j}$ is the lasso estimator defined in example 1 . The DCL is uniformly consistent, and it has a standard normal asymptotic limit and an asymptotically valid uniform confidence band, unlike the lasso and the conservative lasso. These are established in Theorem 3 of Caner and Kock (2018). The formula for the DCL estimator $\hat{b}$ is:

$$
\hat{b}=\hat{\beta}_{C L}+\hat{\Theta} X^{\prime}\left(Y-X \hat{\beta}_{C L}\right) / n
$$

where $\hat{\Theta}$ is an approximate estimate for precision matrix, which will be abstracted away in this paper. Information and detailed properties are described in Section 3.2 of Caner and Kock (2018). Specifically, Caner and Kock (2018) derive the rate of convergence for Wald and $\chi^{2}$ type of tests. They also show that the confidence bands on DCL are contracting at the optimal rate of $n^{-1 / 2}$. By Theorem 2 of Caner and Kock (2018), we have

$$
n^{1 / 2}\left(\hat{b}_{j}-\beta_{0 j}\right)=O_{p}(1)
$$

for $j=1, \cdots, p_{n}$. If we want to test $m$ restrictions on $\beta_{0}$, with $H=\left[h_{1}, \ldots, h_{m}\right]^{\prime}$ representing the restriction matrix of $m \times p_{n}$ dimension, then

$$
\|\mid H\|_{2}=\sqrt{\sum_{i=1}^{m}\left\|h_{i}\right\|_{2}^{2}}=\sqrt{m}
$$

given $\left\|h_{i}\right\|_{2}=1$ in Theorem 2 of Caner and Kock (2018). Conditions C1 and C2 are satisfied. If $m$ is a fixed number, then $H\left(\hat{\beta}-\beta_{0}\right)$ converges to the limit at rate $n^{1 / 2}$, and a $\chi^{2}$ type test converges to the limit at rate $n$ as in (21) of Caner and Kock (2018) 
Example 3. One of the main issues in finance is the analysis of portfolio variance. If we denote the portfolio allocation vector by $w\left(p_{n} \times 1\right)$, and the covariance matrix of asset returns by $\Sigma$, then the portfolio variance is $w^{\prime} \Sigma w$. The out-of-sample estimate of this portfolio variance is $\hat{w}^{\prime} \Sigma \hat{w}$. This estimate can be seen in Ledoit and Wolf (2017) and Ao et al. (2019). The number of assets, $p_{n}$, grows with $n$, which is the time span of the portfolio, and $p_{n}>n$. Let $\operatorname{Eigmax}(A)$ denote the maximum eigenvalue of a matrix $A$ and $C>0$ be a positive constant.

We analyze the global minimum portfolio weights. Define $1_{p_{n}}$ as the $p_{n}$ vector of ones. The weights are computed as

$$
w=\frac{\Sigma^{-1} 1_{p_{n}} / p_{n}}{1_{p_{n}}^{\prime} \Sigma^{-1} 1_{p_{n}} / p_{n}} .
$$

From Theorem 3.3 of Callot et al. (2019), the estimated weights are:

$$
\hat{w}=\frac{\hat{\Theta} 1_{p_{n}} / p_{n}}{1_{p_{n}}^{\prime} \hat{\Theta} 1_{p_{n}} / p_{n}},
$$

where $\hat{\Theta}$ is the nodewise regression estimate of $\Sigma^{-1}$. Take $\beta_{0}=w$, and $\hat{\beta}=\hat{w}$. So our parameter is of dimension $p_{n}$, and it is growing with $n$ and larger than $n$. Our interest centers on the out-of sample portfolio variance estimation, given that we can estimate weights consistently, with a known rate of convergence. First, we start with verifying Condition C1. See that $f(\hat{\beta})=\hat{\beta}^{\prime} \Sigma \hat{\beta}$, and $f\left(\beta_{0}\right)=\beta_{0}^{\prime} \Sigma \beta_{0}$. Condition C2 also holds for this example, and we will show that after verifying Condition C1.

For C1, we have 
$\left\|f\left(\beta_{0}+h\right)-f\left(\beta_{0}\right)-f_{d}\left(\beta_{0}\right) h\right\|_{1}=\left\|\left(\beta_{0}+h\right)^{\prime} \Sigma\left(\beta_{0}+h\right)-\beta_{0}^{\prime} \Sigma \beta_{0}-2 \beta_{0}^{\prime} \Sigma h\right\|_{1}=\left\|h^{\prime} \Sigma h\right\|_{1}$.

Next

$$
\begin{aligned}
\frac{\left\|h^{\prime} \Sigma h\right\|_{1}}{\|h\|_{1}} & =\frac{\left|h^{\prime} \Sigma h\right|}{\|h\|_{1}} \leq \frac{\|h\|_{2}^{2} \operatorname{Eigmax}(\Sigma)}{\|h\|_{1}} \\
& \leq \frac{\|h\|_{2}^{2}}{\|h\|_{2}} \operatorname{Eigmax}(\Sigma)=\|h\|_{2} \operatorname{Eigmax}(\Sigma) \\
& \rightarrow 0 \text { as }\|h\|_{1} \rightarrow 0,
\end{aligned}
$$

where the second inequality follows from $\|h\|_{1} \geq\|h\|_{2}$, and for the convergence to zero we use assumption $\operatorname{Eigmax}(\Sigma) \leq C<\infty$, and the fact that $\|h\|_{1} \rightarrow 0$ implies $\|h\|_{2} \rightarrow 0$. Thus, Condition C1 is satisfied. Then Condition C2 is satisfied since, by Theorem 3.3 of Callot et al. (2019) we have

$$
r_{n}\|\hat{w}-w\|_{1}=O_{p}(1)
$$

where $r_{n}=\frac{\sqrt{n}}{\sqrt{\log p_{n}}} \frac{1}{\bar{s}^{3 / 2}}$, with $\bar{s}=\max _{1 \leq j \leq p} s_{j}$, and $s_{j}$ is the number of nonzero cells in the $j$ th row of the precision matrix. Now, we derive the rate of convergence of the out-of-sample variance estimator. Note that

$$
\left\|f_{d}\left(\beta_{0}\right)\right\|_{1}=\left\|2 \beta_{0}^{\prime} \Sigma\right\|_{1}=2\|\Sigma w\|_{1}
$$

since $\Sigma$ is a $p_{n} \times p_{n}$ symmetric matrix and $\beta_{0}=w$. Define $\sigma_{i, j}$ as the $(i, j)$ th element 
of $\Sigma$ matrix. By (17.3)

$$
\begin{aligned}
\|\Sigma w\|_{1} & \leq\left\|\left|\Sigma\left\|\left.\right|_{1}\right\| w \|_{1}\right.\right. \\
& =\left[\max _{1 \leq j \leq p_{n}} \sum_{i=1}^{p_{n}}\left|\sigma_{i, j}\right|\right]\|w\|_{1} \\
& =\left[\max _{1 \leq j \leq p_{n}} \sum_{i=1}^{p_{n}}\left|\sigma_{i, j}\right|\right] O(\sqrt{\bar{s}}) \\
& =O(\sqrt{\bar{s}}),
\end{aligned}
$$

where the third line uses $\|w\|_{1}=O(\sqrt{\bar{s}})$ by Theorem 3.3 of Callot et al. (2019), and the last line uses the assumption $\left[\max _{1 \leq j \leq p_{n}} \sum_{k=1}^{p_{n}}\left|\sigma_{i, j}\right|\right] \leq C<\infty$. Clearly, we can define $k_{n}:=O\left(2\|\Sigma w\|_{1}\right)$, which implies $k_{n}=O(\sqrt{\bar{s}})$ by the inequality above. Applying Theorem 2.1(b), we obtain a slow rate for the out-of-sample variance estimator compared with the weight estimation

$$
\frac{\sqrt{n}}{\sqrt{\log p_{n}}} \frac{1}{\bar{s}^{2}}\left|\hat{w}^{\prime} \Sigma \hat{w}-w^{\prime} \Sigma w\right|=O_{p}(1)
$$

when $\bar{s}$ is growing with $n$.

\section{Discussion of the Upper Bound}

Here we provide a brief discussion about our results in some econometrics problems. We can see three areas related to our technique that may be beneficial to the researchers. The first area is the many weak instruments literature. In the case of many weak instruments as in Newey and Windmeijer (2009) and Caner (2014), we 
can derive the rate of convergence for the estimation of the sample moments from the estimation of the parameters in the structural equation. If we have $\hat{\beta}$ as the generalized empirical likelihood estimator, with $r_{n}\left\|\hat{\beta}-\beta_{0}\right\|_{2}=O_{p}(1)$, then we can have $r_{n} d_{n}\left\|\hat{g}(\hat{\beta})-\hat{g}\left(\beta_{0}\right)\right\|_{2}=O_{p}(1)$, with

$$
\hat{g}(\hat{\beta})=\frac{1}{n} \sum_{i=1}^{n} Z_{i}\left(y_{i}-x_{i}^{\prime} \hat{\beta}\right),
$$

where $Z_{i}$ is an $m \times 1$ vector of instruments, $m$ is growing with $n$. Let $y_{i}$ be the outcome variable, and $x_{i}$ represent the control and endogenous variables $\left(p_{n} \times 1\right.$ vector). This satisfies our Theorem 2.1(c). The details of this example are provided in the appendix. This extends the results of Example 1 in Section 2 of Caner (2014), and the linear model of Newey and Windmeijer (2009, p. 690-698).

The second one is the portfolio analysis, where we illustrate our results through an example in the main text. Since there are a lot of nonlinear functions in parameters of interest, and it is neither obvious nor trivial to get the rates of these functions in the modern portfolio theory when the number of assets, $p_{n}$, is larger than the time span of the portfolio. Our technique can help. It analyzes the partial derivative of the function at the parameter and automatically finds the rate of convergence through that.

The third area is nonparametric estimation. Our theorem can be applied to obtain the convergence rate of the estimate of the nonparametric function. Consider, for example, the series estimation of the following model

$$
y_{i}=g\left(x_{i}\right)+\varepsilon_{i}, \text { with } E\left(\varepsilon_{i} \mid x_{i}\right)=0,
$$


where the unknown function $g(x)$ can be approximated by a linear combination of basis functions $h^{p_{n}}(x)$ and $p_{n}$ is increasing with $n$. Let $\mathcal{S}$ be the compact support of $x$. Assume that

$$
\sup _{x \in \mathcal{S}}\left|g(x)-h^{p_{n}}(x)^{\prime} \beta\right|=O\left(p_{n}^{-\alpha}\right) \text { for some } \alpha>0 .
$$

Define $\beta^{p_{n}} \equiv \arg \min _{\beta} \sup _{x \in \mathcal{S}}\left|g(x)-h^{p_{n}}(x)^{\prime} \beta\right|$. The estimator of $\beta^{p_{n}}$, denoted by $\hat{\beta}$, is obtained by a linear regression of $y_{i}$ on $h^{p_{n}}\left(x_{i}\right)$. Newey (1997) shows that $\left\|\hat{\beta}-\beta^{p_{n}}\right\|_{2}=O_{p}\left(\sqrt{p_{n}} / \sqrt{n}+p_{n}^{-\alpha}\right)$, which gives the rate for Condition C2. For Condition $\mathrm{C} 1$, consider the linear function $f(\beta)=h^{p_{n}}\left(x_{0}\right)^{\prime} \beta$ for a given $x_{0} \in \mathcal{S}$, so $f\left(\beta^{p_{n}}\right)$ is the approximation of $g\left(x_{0}\right)$ and $f(\hat{\beta})$ is the series estimator $h^{p_{n}}\left(x_{0}\right)^{\prime} \hat{\beta}$. We are interested in the convergence rate of $\left|f(\hat{\beta})-f\left(\beta^{p_{n}}\right)\right|$. Since $f_{d}\left(\beta^{p_{n}}\right)=h^{p_{n}}\left(x_{0}\right)^{\prime}$, Condition C1 holds automatically. By our Theorem 2.1(b) we have

$$
\left|f(\hat{\beta})-f\left(\beta^{p_{n}}\right)\right| \leq \zeta\left(p_{n}\right) \cdot O_{p}\left(\sqrt{\frac{p_{n}}{n}}+\frac{1}{p_{n}^{\alpha}}\right)=O_{p}\left(\zeta\left(p_{n}\right)\left(\sqrt{p_{n}} / \sqrt{n}+p_{n}^{-\alpha}\right)\right)
$$

where $\zeta\left(p_{n}\right)=\sup _{x \in \mathcal{S}}\left\|h^{p_{n}}(x)\right\|_{2} \cdot 1$ Together with (4.1), the rate in (4.2) implies

$\left|h^{p_{n}}\left(x_{0}\right)^{\prime} \hat{\beta}-g\left(x_{0}\right)\right| \leq\left|f(\hat{\beta})-f\left(\beta^{p_{n}}\right)\right|+\sup _{x \in \mathcal{S}}\left|h^{p_{n}}(x)^{\prime} \beta^{p_{n}}-g(x)\right|=O_{p}\left(\zeta\left(p_{n}\right)\left(\sqrt{p_{n}} / \sqrt{n}+p_{n}^{-\alpha}\right)\right)$,

which is the well known convergence rate for the series estimator. Note that our theorem actually gives a sharp upper bound on the convergence rate of the series estimator.

\footnotetext{
${ }^{1}$ Under some regularity conditions, it follows that $\zeta\left(p_{n}\right)=O\left(p_{n}\right)$ for power series and $\zeta\left(p_{n}\right)=$ $O\left(\sqrt{p_{n}}\right)$ for spline series (Corollary 15.1, Li and Racine, 2007).
} 


\section{Simulation}

In this section, we study the degree of conservativeness of the bound derived by Theorem 2.1 via simulation. We consider the lasso estimator discussed in Example 1. The model is generated using (3.1), where $x_{i} \sim \operatorname{iid} N\left(0, I_{p_{n}}\right), u_{i} \sim$ iid $N\left(0, s_{0}\right)$, and $\beta_{0}=\left(\mathbf{1}_{1 \times s_{0}}, 0_{1 \times\left(p_{n}-s_{0}\right)}\right)^{\prime}$. We set $s_{0} \in\{5,10\}, p_{n} \in\{50,100,200,300\}$, and $n \in\{100,200,300\}$. Since $\lambda_{n}=O\left(\sqrt{\log \left(p_{n}\right) / n}\right)$, we select the optimal tuning parameter from the set $\left\{\lambda_{n}=c \sqrt{\log \left(p_{n}\right) / n}, c=0.1,0.25,0.5,1,2,3,4,5,6,7,8,9,10\right\}$ by minimizing the following information criterion,

$$
\lambda^{*}=\arg \min _{\lambda_{n}}\left[\log \hat{\sigma}^{2}\left(\lambda_{n}\right)+\frac{\hat{s}\left(\lambda_{n}\right)}{n} \log (n) \log \log \left(p_{n}\right)\right]
$$

where $\hat{s}\left(\lambda_{n}\right)$ is the number of nonzero entries in the lasso estimator given by (3.2) using tuning parameter $\lambda_{n}$, and $\hat{\sigma}^{2}\left(\lambda_{n}\right)$ is the corresponding mean squared residuals. The term $\log \log \left(p_{n}\right)$ follows the design of Caner, Han and Lee (2018) to deal with the high dimensionality.

We focus on the inference of the first $s_{0}$ parameters in $\beta_{0}$, so $f\left(\beta_{0}\right)=D \beta_{0}$ with $D=\left(I_{s_{0}}, 0\right)$. We compute the ratio $\left\|\left|f_{d}\left(\beta_{0}\right)\left\|\left.\right|_{2}\right\| \hat{\beta}-\beta_{0}\left\|_{2} /\right\| f(\hat{\beta})-f\left(\beta_{0}\right) \|_{2}=\right.\right.$ $s_{0}\left\|\hat{\beta}-\beta_{0}\right\|_{2} /\left\|D \hat{\beta}-D \beta_{0}\right\|_{2}$ to see the tightness of the bound. Table 1 reports the average of this ratio over 1000 replications. It is evident that the averaged ratio is very close to $s_{0}$, which implies that most of the zero elements in $\beta_{0}$ are estimated as zero by the lasso. In the (infeasible) oracle case where all zero parameters are exactly estimated as zero, the ratio would be equal to $s_{0}$. We clearly see that our upper bound grows with sparsity, and will not be that tight unless the model is 
very sparse. However, the main problem is that in high dimensional econometrics, it is complicated to get closed-form solutions; hence we rely on upper bounds. For example, the oracle inequalities, $l_{1}$ norm results are all upper bounded.

Table 1: The Ratio of the Upper Bound to the Function of the Lasso Estimator

\begin{tabular}{|c|c|c|c|c|}
\hline$n, s_{0}$ & $p_{n}=50$ & $p_{n}=100$ & $p_{n}=200$ & $p_{n}=300$ \\
\hline \hline 100,5 & 5.23 & 5.09 & 5.04 & 5.02 \\
\hline 200,5 & 5.20 & 5.11 & 5.06 & 5.05 \\
\hline 300,5 & 5.15 & 5.08 & 5.06 & 5.04 \\
\hline 100,10 & 10.26 & 10.11 & 11.64 & 13.40 \\
\hline 200,10 & 10.45 & 10.20 & 10.09 & 10.07 \\
\hline 300,10 & 10.41 & 10.22 & 10.13 & 10.10 \\
\hline
\end{tabular}

\section{Conclusion}

We provide an upper bound for the functions of the estimators in high dimensions. We also show three examples to illustrate the main theorem. It is possible to extend our result to more relevant econometrics issues in the age of big data. In summary, our method can be useful for obtaining the rate of convergence of functionals of estimators, since it uses the partial derivative of the function at $\beta_{0}$. Our bound can be beneficial in high dimensional scenarios where it may be difficult to have direct proof of the rate of convergence. 


\section{REFERENCES}

Abadir, K. and J.R. Magnus (2005). Matrix Algebra. Cambridge University Press. Cambridge.

Ao, M., Y.Li and X. Zheng (2019). Approaching mean-variance efficiency for large portfolios. Review of Financial Studies, Forthcoming.

Buhlmann, P. and S. van de Geer (2011). Statistics for High-Dimensional Data. Springer Verlag, Berlin.

Callot, L., Caner, M., O, Onder, E. Ulasan (2019). A nodewise regression approach to estimating large portfolios. Journal of Business and Economic Statistics, Forthcoming.

Caner, M. (2014). Near Exogeneity and Weak Identification in Generalized Empirical Likelihood Estimators. Journal of Econometrics, 182, 247-288.

Caner, M, and X. Han, and Y. Lee (2018). Adaptive Elastic Net GMM Estimation with Many Invalid Moment Conditions: Simultaneous Model and Moment Selection. Journal of Business and Economics Statistics,36, 24-46.

Caner, M. and A.B. Kock. (2018). Asymptotically Honest Confidence Regions for High Dimensional Parameters by the Desparsified Conservative Lasso. Journal of Econometrics, 203, 143-168.

Fan, J., Y. Liao, and X. Shi (2015). Risks of large portfolios. Journal of Econometrics, 186, 367-387.

Hastie, T., R. Tibshirani, and J. Friedman (2009). The Elements of Statistical Learning. Springer Verlag. NYC.

Horn, R.A. and C. Johnson (2013). Matrix Analysis. Second Edition. Cambridge 
University Press, Cambridge.

Ledoit, O., M. Wolf (2017). Nonlinear shrinkage of the covariance matrix for portfolio selection: Markowitz meets Goldilocks. Review of Financial Studies, 30, $4349-4388$.

Li, Q. and J. Racine (2007). Nonparametric Econometrics: Theory and Practice. Princeton University Press.

Newey, W, (1997). Convergence Rates and Asymptotic Normality for Series Estimators. Journal of Econometrics, 79(1), 147-168.

Newey, W, and F. Windmeijer (2009). Generalized Method of Moments with Many Weak Moment Conditions. Econometrica, 77, 687-719.

Tibshirani, R. (1996). Regression Shrinkage and Selection via the Lasso. Journal of the Royal Statistical Society Series B, 58, 267-288.

Van de Geer, S., P. Buhlmann, Y. Ritov, and R. Dezeure (2014). On asymptotically optimal confidence regions and test for high-dimensional models. The Annals of Statistics, 42, 1166-1202.

Van der Vaart, A.W. (2000). Asymptotic Statistics. Cambridge University Press, Cambridge.

\section{Appendix}

The appendix has three parts. In Part A, we introduce the matrix inequalities that are used in the proofs. In Part B, we provide the proof of our theorem and show why a classical proof of the delta method in high dimensions does not work. In Part 
$\mathrm{C}$, we give more examples that are tied to our main theorem.

\section{PART A.}

Take a generic matrix, $A$, which is of dimension $m \times p_{n}$. Denote the Frobenius

norm for a matrix as $\left\||A \||_{2}=\sqrt{\sum_{i=1}^{m} \sum_{j=1}^{p_{n}} a_{i j}^{2}}\right.$. Note that in some literature such as Horn and Johnson (2013), this definition is not considered a matrix norm, due to the lack of submultiplicativity. However, our results will not change regardless of matrix norm definitions. If we use Horn and Johnson (2013) definitions, our results can be summarized in an algebraic form, rather than the matrix norm format. Define

$$
A=\left[\begin{array}{c}
a_{1}^{\prime} \\
\vdots \\
a_{m}^{\prime}
\end{array}\right],
$$

where $a_{i}$ is a $p_{n} \times 1$ vector, and its transpose is $a_{i}^{\prime}, i=1, \cdots, m$. Then for a generic $p_{n} \times 1$ vector $x$,

$$
\|A x\|_{2}=\sqrt{\sum_{i=1}^{m}\left(a_{i}^{\prime} x\right)^{2}} \leq\left(\sqrt{\sum_{i=1}^{m}\left\|a_{i}\right\|_{2}^{2}}\right)\|x\|_{2}=\|\mid A\|\left\|_{2}\right\| x \|_{2},
$$

where the inequality is obtained by the Cauchy-Schwarz inequality. Note that if we apply Horn and Johnson's (2013) norm definition, this matrix norm inequality still 
holds, but we cannot use the matrix norm. In that case we have

$$
\|A x\|_{2} \leq\left(\sqrt{\sum_{i=1}^{m}\left\|a_{i}\right\|_{2}^{2}}\right)\|x\|_{2} .
$$

Also, see that the results (2.1)-(2.3) can be obtained in other matrix norms. A simple Holder's inequality provides

$$
\|A x\|_{1} \leq\left\|\left|A\left\|\left.\right|_{1}\right\| x \|_{1}\right.\right.
$$

where we define the maximum column sum matrix norm: $\|A\| \|_{1}=$ $\max _{1 \leq j \leq p_{n}} \sum_{i=1}^{m}\left|a_{i j}\right|$, and $a_{i j}$ is the $(i, j)$ th element of the matrix $A$. Theorem 2.1 can be written in $l_{1}$ norm replacing $l_{2}$ norm. We can also extend these results to another norm. A simple inequality provides

$$
\|A x\|_{\infty} \leq\left\|\left|A\left\|\left.\right|_{\infty}\right\| x \|_{\infty}\right.\right.
$$

where we define the maximum row sum matrix norm: $\left\||A \||_{\infty}=\max _{1 \leq i \leq m} \sum_{j=1}^{p_{n}}\left|a_{i j}\right|\right.$ and Theorem 2.1 can be written in $l_{\infty}$ norm as well.

PART B.

First, we show why the classical proof of the delta method does not work in high 
dimensions. However, this is not a negative result since it guides us towards the solution.

First by Condition C1, via p.352 of Abadir and Magnus (2005) $l():. D \subset R^{p_{n}} \rightarrow$ $R^{m}$ is a vector function

$$
\left\|f(\hat{\beta})-f\left(\beta_{0}\right)-f_{d}\left(\beta_{0}\right)\left[\hat{\beta}-\beta_{0}\right]\right\|_{2}=\left\|l\left(\hat{\beta}-\beta_{0}\right)\right\|_{2} .
$$

and

$$
\left\|l\left(\hat{\beta}-\beta_{0}\right)\right\|_{2}=o_{p}\left(\left\|\hat{\beta}-\beta_{0}\right\|_{2}\right)
$$

where we use Lemma 2.12 of van der Vaart (2000).

Since we are given $r_{n}\left\|\hat{\beta}-\beta_{0}\right\|_{2}=O_{p}(1)$ which is Condition $\mathrm{C} 2$, by (7.6)

$$
r_{n}\left\|l\left(\hat{\beta}-\beta_{0}\right)\right\|_{2}=o_{p}(1)
$$

By (7.5) $-(7.7)$

$$
\left\|r_{n}\left[f(\hat{\beta})-f\left(\beta_{0}\right)\right]-r_{n}\left[f_{d}\left(\beta_{0}\right)\right]\left[\hat{\beta}-\beta_{0}\right]\right\|_{2}=o_{p}(1)
$$

But this is the same result as in the regular delta method. (7.8) is mainly a simple extension of Theorem 3.1 in van der Vaart (2000) to Euclidean spaces so far. However, the main caveat comes from the derivative matrix $f_{d}\left(\beta_{0}\right)$, which is of dimension $m \times p_{n}$. The rate of the matrix plays a role when $p_{n} \rightarrow \infty$ as $n \rightarrow \infty$. For example, both $r_{n}\left[f_{d}\left(\beta_{0}\right)\right]\left[\hat{\beta}-\beta_{0}\right]$ and $r_{n}\left[f(\hat{\beta})-f\left(\beta_{0}\right)\right]$ may be diverging, but $r_{n}\left\|\hat{\beta}-\beta_{0}\right\|_{2}=$ $O_{p}(1)$. Hence the delta method is not that useful if our interest centers on getting 
rates for estimators as well as functions of estimators that converge. In the fixed $p$ case, this is not an issue, since the matrix derivative will not affect the rate of convergence at all, as long as this is bounded away from zero, and is bounded from above away from infinity. Note that boundedness assumptions may not be intact when we have $p_{n} \rightarrow \infty$, as $n \rightarrow \infty$. Next part shows how to correct this problem.

Proof of Theorem 2.1. From Condition C1, using p.352 of Abadir and Magnus (2005), or proof of Theorem 3.1 in van der Vaart (2000) yields

$$
f(\hat{\beta})-f\left(\beta_{0}\right)=f_{d}\left(\beta_{0}\right)\left[\hat{\beta}-\beta_{0}\right]+l\left(\hat{\beta}-\beta_{0}\right) .
$$

Using the Euclidean norm and the triangle inequality, we have

$$
\begin{aligned}
\left\|f(\hat{\beta})-f\left(\beta_{0}\right)\right\|_{2} & =\left\|f_{d}\left(\beta_{0}\right)\left[\hat{\beta}-\beta_{0}\right]+l\left(\hat{\beta}-\beta_{0}\right)\right\|_{2} \\
& \leq\left\|f_{d}\left(\beta_{0}\right)\left[\hat{\beta}-\beta_{0}\right]\right\|_{2}+\left\|l\left(\hat{\beta}-\beta_{0}\right)\right\|_{2}
\end{aligned}
$$

Next, multiply each side by $r_{n}$, and use (7.7)

$$
r_{n}\left\|f(\hat{\beta})-f\left(\beta_{0}\right)\right\|_{2} \leq r_{n}\left\|f_{d}\left(\beta_{0}\right)\left[\hat{\beta}-\beta_{0}\right]\right\|_{2}+o_{p}(1)
$$

Then apply the matrix norm inequality in (17.1) to the first term on the right side of (7.9)

$$
r_{n}\left\|f_{d}\left(\beta_{0}\right)\left[\hat{\beta}-\beta_{0}\right]\right\|_{2} \leq r_{n}\left[\left\|\mid f_{d}\left(\beta_{0}\right)\right\|_{2}\right]\left[\left\|\hat{\beta}-\beta_{0}\right\|_{2}\right]
$$


Substitute (7.10) into (7.9) to have

$$
r_{n}\left\|f(\hat{\beta})-f\left(\beta_{0}\right)\right\|_{2} \leq r_{n}\left[\left\|\mid f_{d}\left(\beta_{0}\right)\right\|_{2}\right]\left[\left\|\hat{\beta}-\beta_{0}\right\|_{2}\right]+o_{p}(1)
$$

In (7.11) on the right side note that by Condition C2, we have $r_{n}\left\|\hat{\beta}-\beta_{0}\right\|_{2}=O_{p}(1)$.

$$
r_{n}\left\|f(\hat{\beta})-f\left(\beta_{0}\right)\right\|_{2} \leq\left[\left\|\mid f_{d}\left(\beta_{0}\right)\right\|_{2}\right] O_{p}(1)+o_{p}(1)
$$

Next, divide each side by $r_{n}$ to have

$$
\left\|f(\hat{\beta})-f\left(\beta_{0}\right)\right\|_{2} \leq\left[\left\|\mid f_{d}\left(\beta_{0}\right)\right\|_{2}\right] O_{p}\left(\frac{1}{r_{n}}\right)+o_{p}\left(\frac{1}{r_{n}}\right) .
$$

Then parts a)-c) follow through by substituting the different specifications for $\left[\left\|\mid f_{d}\left(\beta_{0}\right)\right\|_{2}\right]$ on the right side.

Note that we can prove Theorem 2.1 in $l_{1}$ and $l_{\infty}$ norms, too. To see this, (7.6)(17.7) also hold with $l_{1}$ and $l_{\infty}$ norms. This is true since Lemma 2.12 of van der Vaart (2000) holds also with $l_{1}$ and $l_{\infty}$ norms. Hence, the proof will follow with $l_{1}$ and $l_{\infty}$ versions of $\mathrm{C} 1$ and $\mathrm{C} 2$ with (7.3) and (7.4).

\section{Q.E.D.}

Remark. Note that the proof of Theorem 2.1 mainly uses triangle and CauchySchwarz inequalities. Under some special conditions, these inequalities hold with the equality sign. For example, $\left|a^{\prime} b\right|=\|a\|_{2}\|b\|_{2}$ and $\|a+b\|_{2}=\|a\|_{2}+\|b\|_{2}$ when $a$ and $b$ are on the same ray. However, these conditions are very restrictive and they do not hold in general. Thus, we only have inequalities in most cases. 
PART C.

Example AC.1. Here we use the setup on p.690 and p.698 of Newey and Windmeijer (2009), which is also used in section 2 of Caner (2014). For $i=1, \cdots, n$

$$
\begin{gathered}
y_{i}=x_{i}^{\prime} \beta_{0}+\epsilon_{i}, \\
x_{i}=\Psi_{i}+\eta_{i},
\end{gathered}
$$

and we have $E\left[\epsilon_{i} \mid Z_{i}, \Psi_{i}\right]=0, E\left[\eta_{i} \mid Z_{i}, \Psi_{i}\right]=0 . \quad \eta_{i}, \epsilon_{i}$ are correlated. Also $y_{i}$ is a scalar, $x_{i}$ is a $p \times 1$ vector of control and endogenous variables, and $p$ is fixed here. $Z_{i}$ is an $m \times 1$ vector of instrumental variables. $\Psi_{i}$ is a $p \times 1$ vector of reduced form values. The moment function is an $m \times 1$ vector, where $m$ grows with $n$, for $\beta$ in a compact subset of $R^{p}$ :

$$
\hat{g}(\beta)=\frac{1}{n} \sum_{i=1}^{n} Z_{i}\left(y_{i}-x_{i}^{\prime} \beta\right) .
$$

First, define the sequence $\mu_{n}$ such that $\mu_{n}=o\left(n^{1 / 2}\right)$. This will indicate less than strong identification. Suppose it has the reduced form

$$
\begin{gathered}
x_{i}=\left(z_{1 i}^{\prime}, x_{2 i}^{\prime}\right)^{\prime}, \\
x_{2 i}=\pi_{21} z_{1 i}+\frac{\mu_{n}}{n^{1 / 2}} z_{2 i}+\eta_{2 i}, \\
Z_{i}=\left(z_{1 i}^{\prime}, Z_{2 i}^{\prime}\right)^{\prime},
\end{gathered}
$$

where $z_{1 i}$ is a $p_{1}$ vector of included exogenous variables (controls), $z_{2 i}$ is a ( $p-$ 
$\left.p_{1}\right) \times 1$ vector of excluded exogenous variables, and $Z_{2 i}$ is an $\left(m-p_{1}\right) \times 1$ vector of instruments. If $z_{2 i}$ is not observed, this is approximated by $Z_{2 i}$ which can be a power series or splines. Define a $p \times p$ matrix

$$
\tilde{S}_{n}=\left[\begin{array}{cc}
I_{p_{1}} & 0 \\
\pi_{21} & I_{p-p_{1}}
\end{array}\right]
$$

Define $S_{n}=\tilde{S}_{n} \operatorname{diag}\left(\mu_{1 n}, \cdots, \mu_{p_{1}, n}, \mu_{p_{1}+1, n}, \cdots, \mu_{p, n}\right)$ where the $\operatorname{diag}()$ is a $p \times p$ diagonal matrix, its first $p_{1}$ elements are $n^{1 / 2}$, and the rest is $\mu_{n}$. So $\mu_{j, n}=n^{1 / 2}$ for $j=1, \cdots, p_{1}$ and $\mu_{j, n}=\mu_{n}$ for $j=p+1, \cdots, p$. The reduced form can be written as:

$$
\Psi_{i}=\left(\begin{array}{c}
Z_{1 i} \\
\pi_{21} z_{1 i}+\frac{\mu_{n}}{n^{1 / 2}} z_{2 i}
\end{array}\right)=\frac{S_{n} z_{i}}{n^{1 / 2}}
$$

Note that

$$
\hat{g}(\hat{\beta})-\hat{g}\left(\beta_{0}\right)=\frac{1}{n} Z_{i} x_{i}^{\prime}\left(\hat{\beta}-\beta_{0}\right)
$$

as in supplement (Appendix) p.11 of the proof of Theorem 2 in Newey and Windmeijer (2009). Condition C1 is not needed since the system is linear in $\beta$. There is no need for (7.6). We benefit from a direct proof of Theorem 2.1, By Theorem 3 of Newey and Windmeijer (2009)

$$
\left\|S_{n}^{\prime}\left(\hat{\beta}-\beta_{0}\right)\right\|_{2}=O_{p}(1)
$$


which is Condition C2. Next with $f_{d}\left(\beta_{0}\right)=\hat{G}=\frac{-1}{n} \sum_{i=1}^{n} Z_{i} x_{i}^{\prime}$. So clearly

$$
\left\|\hat{g}(\hat{\beta})-\hat{g}\left(\beta_{0}\right)\right\|_{2}=\left\|\hat{G}\left(\hat{\beta}-\beta_{0}\right)\right\|_{2}
$$

Then

$$
\begin{aligned}
n^{1 / 2} \|\left(\hat{g}(\hat{\beta})-\hat{g}\left(\beta_{0}\right) \|_{2}\right. & =\left\|\hat{G} n^{1 / 2} S_{n}^{-1^{\prime}} S_{n}^{\prime}\left(\hat{\beta}-\beta_{0}\right)\right\|_{2} \\
& \leq\left\|\hat{G} n^{1 / 2} S_{n}^{-1^{\prime}}\right\|_{2}\left\|S_{n}^{\prime}\left(\hat{\beta}-\beta_{0}\right)\right\|_{2} \\
& =O_{p}(1) O_{p}(1),
\end{aligned}
$$

by the proof of Lemma A.7 in Newey and Windmeijer (2009), we have $\left\|\hat{G} n^{1 / 2} S_{n}^{-1^{\prime}}\right\|_{2}=O_{p}(1)$, and the other rate is by (17.14). Since $S_{n}$ has mixed rates, $n^{1 / 2}$ and $\mu_{n}$, we find that $n^{1 / 2}$ is faster or equal to them, satisfying Theorem 2.1 .

\section{Example AC.2.}

Example 3 in the main text considers the out-of-sample portfolio variance using the global minimum portfolio weights. Alternatively, we can apply Theorem 2.1 to study the convergence rate of the estimated portfolio variance using an estimated matrix $\hat{\Sigma}$ and a given weight $w$. Hence, in this example we are interested in the parameter $\beta_{0}=\operatorname{vech}\left(\Sigma_{0}\right)$ and the function

$$
f\left(\beta_{0}\right)=w^{\prime} \Sigma_{0} w=\left(w^{\prime} \otimes w^{\prime}\right) D_{p} \operatorname{vech}\left(\Sigma_{0}\right)=\left(w^{\prime} \otimes w^{\prime}\right) D_{p} \beta_{0},
$$

where $\Sigma_{0}$ is $q \times q, w$ is $q \times 1, \beta_{0}$ is $p_{n} \times 1$ with $p_{n}=q(q+1) / 2$, and $D_{p}$ is a duplication matrix. Let $q$ (and thus $p_{n}$ ) be growing with $n$. Since $f(\beta)$ is linear in $\beta$, Condition 
$\mathrm{C} 1$ is always satisfied. Recall that $\|A\|_{\infty}=\max _{i} \max _{j}\left|A_{i j}\right|$ of the matrix $\mathrm{A}$, (note that this is different than $\left\||A \||_{\infty}\right.$ which is the maximum row-sum matrix norm), $\|\mid A\|_{2}$ is the Frobenius norm of matrix $\mathrm{A},\|v\|_{2}$ is the $l_{2}$ norm of a vector $v$.

For Condition C2, van de Geer et al. (2014) we have

$$
\|\hat{\Sigma}-\Sigma\|_{\infty}=O_{p}\left(\sqrt{\frac{\log q}{n}}\right)
$$

by the symmetry of $\hat{\Sigma}-\Sigma$, so by p.365 of Horn and Johnson (2013)

$$
\|\mid \hat{\Sigma}-\Sigma\|\left\|_{2} \leq q\right\| \hat{\Sigma}-\Sigma \|_{\infty}=O_{p}\left(q \sqrt{\frac{\log q}{n}}\right),
$$

which implies

$$
\left\|\hat{\beta}-\beta_{0}\right\|_{2} \leq\|\mid \hat{\Sigma}-\Sigma\| \|_{2} \leq O_{p}\left(q \sqrt{\frac{\log q}{n}}\right) .
$$

Hence, we require that $q$ has to diverge at a slower rate than $n$ for $\mathrm{C} 2$ to hold.

Next

$$
\begin{aligned}
\left\|\mid f_{d}\right\|_{2} & =\left\|\mid\left(w^{\prime} \otimes w^{\prime}\right) D_{p}\right\|_{2}=\operatorname{trace}\left[\left(w^{\prime} \otimes w^{\prime}\right) D_{p} D_{p}^{\prime}(w \otimes w)\right]^{1 / 2} \\
& \leq \operatorname{Eigmax}\left(D_{p} D_{p}^{\prime}\right)^{1 / 2}\left\|w^{\prime} \otimes w^{\prime}\right\|_{2} \\
& =\sqrt{2}\left\|w^{\prime} \otimes w^{\prime}\right\|_{2}=\sqrt{2}\|w\|_{2}^{2} \leq \sqrt{2}\|w\|_{1}^{2},
\end{aligned}
$$

where the third line uses the fact that $\operatorname{Eigmax}\left(D_{p} D_{p}^{\prime}\right)=\operatorname{Eigmax}\left(D_{p}^{\prime} D_{p}\right)=2$ because $D_{p}^{\prime} D_{p}$ is a diagonal matrix with elements 1 and 2. If we assume that $\|w\|_{1}^{2}=k_{n}$, 
i.e., the gross exposure is of order $k_{n}^{1 / 2}$, then

$$
f(\hat{\beta})-f\left(\beta_{0}\right)=w^{\prime} \hat{\Sigma} w-w^{\prime} \Sigma_{0} w=O_{p}\left(k_{n} q \sqrt{\frac{\log q}{n}}\right)
$$

by Theorem 2.1(b).

Note that by using tools in modern high dimensional portfolio analysis as in Example 3 with a longer proof, we can get a rate better than the one in (7.15). Here, we compare the estimator's rate with the function of the estimator, which is the portfolio variance estimation by fixed weights. In this example, it turns out that the gross exposure is the key to the rate.

Now we provide some perspective in the following remark why our proof technique is desirable.

Remark. This remark shows a directly derivation of the bound for Example 3 in the main text without using technique we propose. Compared to the proposed upper bound technique, the following alternative method involves more steps and more comparisons between terms and matrix inequalities. First, arranging the terms in the out-of-sample variance yields

$$
\begin{aligned}
\left|f(\hat{\beta})-f\left(\beta_{0}\right)\right| & =\left|\hat{\beta}^{\prime} \Sigma \hat{\beta}-\beta_{0}^{\prime} \Sigma \beta_{0}\right|=\left|\hat{w}^{\prime} \Sigma \hat{w}-w^{\prime} \Sigma w\right| \\
& =\left|\hat{w}^{\prime} \Sigma \hat{w}-w^{\prime} \Sigma \hat{w}+w^{\prime} \Sigma \hat{w}-w^{\prime} \Sigma w\right| \\
& =\left|(\hat{w}-w)^{\prime} \Sigma \hat{w}+w^{\prime} \Sigma(\hat{w}-w)\right| \\
& =\left|(\hat{w}-w)^{\prime} \Sigma \hat{w}-(\hat{w}-w)^{\prime} \Sigma w+(\hat{w}-w)^{\prime} \Sigma w+w^{\prime} \Sigma(\hat{w}-w)\right| \\
& =\left|(\hat{w}-w)^{\prime} \Sigma(\hat{w}-w)+2(\hat{w}-w)^{\prime} \Sigma w\right|,
\end{aligned}
$$


where we add and subtract $w^{\prime} \Sigma \hat{w}$ and $(\hat{w}-w)^{\prime} \Sigma w$ to get the third and the last equalities, respectively, and we use symmetricity of $\Sigma$ in the last equality.

Using the above equality, we have

$$
\begin{aligned}
\left|f(\hat{\beta})-f\left(\beta_{0}\right)\right| & =\left|(\hat{w}-w)^{\prime} \Sigma(\hat{w}-w)+2(\hat{w}-w)^{\prime} \Sigma w\right| \\
& \leq\left|(\hat{w}-w)^{\prime} \Sigma(\hat{w}-w)\right|+2\left|(\hat{w}-w)^{\prime} \Sigma w\right| \\
& \leq\|\hat{w}-w\|_{1}\|\Sigma(\hat{w}-w)\|_{\infty}+2\|\hat{w}-w\|_{1}\|\Sigma w\|_{\infty} \\
& \leq\|\hat{w}-w\|_{1}\|\| \Sigma\left\|_{\infty}\right\| \hat{w}-w\left\|_{1}+2\right\| \hat{w}-w\left\|_{1}\right\| \Sigma\left\|_{\infty}\right\| w \|_{1} \\
& =\|\hat{w}-w\|_{1}^{2}\|\Sigma\|_{\infty}+2\|\hat{w}-w\|_{1}\|\Sigma\|_{\infty}\|w\|_{1}
\end{aligned}
$$

where the second line uses the triangle inequality, the third line uses Holder's inequality, the fourth line follows from $\|A x\|_{\infty} \leq\|A\|_{\infty}\|x\|_{1}$ for a generic $A$ matrix and a generic vector $x$ by our definition of $\|A\|_{\infty}$.

Next, we evaluate the first term in (7.18). By Theorem 3.3 of Callot et al. (2019), we have

$$
\|\hat{w}-w\|_{1}=O_{p}\left(\frac{\sqrt{\log p_{n}}}{\sqrt{n}} \bar{s}^{3 / 2}\right) .
$$

We assume then $\|\Sigma\|_{\infty}=O(1)$. So the first term's rate is:

$$
\|\hat{w}-w\|_{1}^{2}\|\Sigma\|_{\infty}=O_{p}\left(\frac{\sqrt{\log p_{n}}}{\sqrt{n}} \bar{s}^{3 / 2}\right) .
$$

Then since $\|w\|_{1}=O\left(\bar{s}^{1 / 2}\right)$ by Remark in Theorem 3.3 of Callot et al. (2019), the 
second term on the right side of (7.18) has the rate

$$
2\|\hat{w}-w\|_{1}\|\Sigma\|_{\infty}\|w\|_{1}=O_{p}\left(\frac{\sqrt{\log p_{n}}}{\sqrt{n}} \bar{s}^{3 / 2}\right) O_{p}\left(\bar{s}^{1 / 2}\right)=O_{p}\left(\frac{\sqrt{\log p_{n}}}{\sqrt{n}} \bar{s}^{2}\right) .
$$

So (7.20) rate is slower than (7.19), hence it will be the rate of convergence for the out-of-sample variance estimator. This means that $r_{n}=\frac{\sqrt{\log p_{n}}}{\sqrt{n}} \frac{1}{\bar{s}^{3 / 2}}$ and $k_{n}=\bar{s}^{1 / 2}$. By Theorem 2.1(b), $r_{n} / k_{n}=\frac{\sqrt{\log p_{n}}}{\sqrt{n}} \frac{1}{\bar{s}^{2}}$. Thus, we reach the same conclusion as in Example 3, but with additional inequalities and comparisons. Of course, there is one caveat, the assumptions are slightly different, in example 3 we assume that $\|\mid \Sigma\| \|_{1}$ is finite, whereas in the remark here we assume that $\|\Sigma\|_{\infty}$ is finite. In higher dimensions, the former can be stronger than the latter depending on the sparsity of columns of $\Sigma$.

Next we provide a remark that measures the divergence between two norms that are used.

Remark. The convergence rate implied by Theorem 2.1 might be potentially conservative under some cases. Whether the rate is sharp or conservative depends on the specific setup of the estimator of interest. To see whether our theorem gives a conservative rate in Example 3, we can develop a measure of divergence between our upper bound and the one derived in (7.20) from the direct proof in the previous remark. Let div represent the divergence between two norms of $\Sigma$

$$
d i v=\frac{\|\mid \Sigma\|_{1}}{\|\Sigma\|_{\infty}}=\frac{\max _{1 \leq j \leq p_{n}} \sum_{i=1}^{p_{n}}\left|\sigma_{i, j}\right|}{\max _{1 \leq j \leq p_{n}} \max _{1 \leq i \leq p_{n}}\left|\sigma_{i, j}\right|}
$$

We can see from the divergence measure that our upper bound can be quite large in certain cases, such as in the case where $\sigma_{i, j}$ 's are constants and bounded away from 
zero and infinity. But as said earlier, in case of $\|\mid \Sigma\| \|_{1} \leq C<\infty$, with $\mathrm{C}$ being a positive constant, the rate is given by our theorem is not conservative. 